ZU-TH 3/95

February 1995

hep-th/9502045

\title{
The number of sphaleron instabilities of the Bartnik-McKinnon solitons and non-Abelian black holes
}

\author{
M.S. Volkov, [ O. Brodbeck, G. Lavrelashvili, ] and N. Straumann \\ Institut für Theoretische Physik der Universität Zürich-Irchel, Winterthurerstrasse 190, \\ CH-8057 Zürich, \\ Switzerland
}

\begin{abstract}
It is proven that there are precisely $n$ odd-parity sphaleron-like unstable modes of the $n$-th Bartnik-McKinnon soliton and the $n$-th non-abelian black hole solution of the Einstein-Yang-Mills theory for the gauge group $S U(2)$.
\end{abstract}

\footnotetext{
${ }^{1}$ On leave from Physical-Technical Institute of the Academy of Sciences of Russia, Kazan 420029, Russia

${ }^{2}$ On leave of absence from Tbilisi Mathematical Institute, 380093 Tbilisi, Georgia
} 


\section{Introduction}

It is by now well known that Bartnik-McKinnon (BK) solitons [1] and non-abelian black holes [2] of the $S U(2)$ Einstein-Yang-Mills (EYM) theory are very fragile objects. A linear perturbation analysis [3] showed that these solutions are unstable and non-linear numerical studies [4], [5] revealed that the instability is quite dramatic.

The unstable modes found in [3]-[5] belong to perturbations within the original BK ansatz. This type of instabilities will be referred to as "gravitational", because they have no flat spacetime analogues. The numerical results for the few lowest members of the solution families led to the suspicion that the $n$-th EYM (soliton or black hole) solution has exactly $n$ such unstable gravitational modes, a conjecture which is still not proven.

Beside these even-parity modes there is a second class of exponentially growing modes, with the opposite parity, which we call "sphaleron-like" instabilities, because they have a similar origin as for the sphaleron solution of the Yang-Mills-Higgs system in flat spacetime. Heuristically, this type of instabilities is related to the existence of non-contractible loops in configuration space, passing through the equilibrium solution (at least for $n$ odd) [6]. This fact suggests that the latter are some kind of saddle points. For odd $n$ 's there are also paths connecting homotopically distinct vacua, differing in their Chern-Simons number, which pass through the equilibrium solutions [7], [8]. It has been shown analytically that there exists at least one sphaleron-like unstable mode for each member of the BK family and this remains true for "generic" solitons belonging to general gauge groups [9], [10]. The same has been shown for the $S U(2)$ EYM black holes [11] and "generic" non-abelian black holes for any compact gauge group [10]. Numerical studies [12] for the few lowest members of the BK family again suggests that there are also exactly $n$ sphaleron-like instabilities. It is the main purpose of the present paper to prove this conjecture, both for BK solitons and non-abelian black holes.

Our demonstration is based on the study of some "dual" pairs of Schrödinger equations, whose partners are formally related in the same manner as in supersymmetric quantum mechanics. Thanks to a residual gauge group, it is possible to find the zeroenergy solutions in sufficiently explicit form to read off the number of their nodes and hence, for an appropriate choice of the pair, the number of bound states, i.e., unstable modes. We find it quite remarkable that this can be deduced, in spite of the fact that the BK solutions and their black hole counterparts are not known analytically. (Rigorous existence proof have been given in [13], [14], [15]).

\section{Basic equations}

The basic equations for spherically symmetric $S U(2)$ EYM fields, as well as for spherical perturbations of the EYM solitons and black holes, have been derived before (even for general gauge groups [9]). We follow the notations in Ref.[16], where the time-dependent spherically-symmetric gravitational and gauge fields are parameterized as follows.

For the metric we use Schwarzschild coordinates

$$
d s^{2}=-N S^{2} d t^{2}+N^{-1} d r^{2}+r^{2}\left(d \vartheta^{2}+\sin ^{2} \vartheta d \varphi^{2}\right)
$$

and the $S U(2)$ gauge potential is represented as

$$
A=a_{0} \tau_{r} d t+a_{1} \tau_{r} d r+(w-1)\left(\tau_{\varphi} d \vartheta-\tau_{\vartheta} \sin \vartheta d \varphi\right)+\tilde{w}\left(\tau_{\vartheta} d \vartheta+\tau_{\varphi} \sin \vartheta d \varphi\right) .
$$


The metric coefficients $N, S$ and gauge amplitudes $a_{0}, a_{1}, w, \tilde{w}$ are functions of $r$ and $t$, and $\tau_{r}, \tau_{\vartheta}, \tau_{\varphi}$ denote the spherical generators of $S U(2)$, normalized such that $\left[\tau_{r}, \tau_{\vartheta}\right]=\tau_{\varphi}$, etc.

The coupled EYM field equations, expressed in terms of the functions in (11) and (2), can be found in 16. In the static case, with $a_{0}=a_{1}=\tilde{w}=0$, these equations are known to admit soliton and black hole solutions [1], [2], characterized by the number of nodes of the gauge amplitude $w(r)$. Here we repeat only the resulting perturbation equations for these equilibrium solutions [3], [11], [16].

Since the parity transformation, $\vartheta \rightarrow \pi-\vartheta, \varphi \rightarrow \varphi+\pi$, is a symmetry operation, the even-parity perturbations $(\delta N, \delta S, \delta w)$ and the odd-parity modes $\left(\delta a_{0}, \delta a_{1}, \delta \tilde{w}\right)$ decouple. The perturbation equation for the even-parity ("gravitational") modes can be brought into the form of a $P$-wave Schrödinger equation [3]

$$
-\eta^{\prime \prime}+\left(S^{2} N \frac{3 w^{2}-1}{r^{2}}+2\left(\frac{S^{\prime}}{S}\right)^{\prime}\right) \eta=\omega^{2} \eta,
$$

where $\delta w=\eta(\rho) \exp (i \omega t)$, and a prime always denotes differentiation with respect to the radial coordinate $\rho$ defined by

$$
\frac{d \rho}{d r}=\frac{1}{N S}
$$

$(\rho(0)=0$ for solitons and $\rho=-\infty$ at the horizon for black holes).

For the odd-parity ("sphaleron") modes we choose the temporal gauge, $\delta a_{0}=0$, and adopt the following notations for the perturbation amplitudes $\delta a_{1}, \delta \tilde{w}$ :

$$
\delta a_{1}=\alpha(\rho) \exp (i \omega t), \quad \delta \tilde{w}=\xi(\rho) \exp (i \omega t) .
$$

For $\alpha$ and $\xi$ we obtain the coupled system, consisting of the second order equation

$$
-\xi^{\prime \prime}+\frac{N S^{2}}{r^{2}}\left(w^{2}-1\right) \xi+N S w^{\prime} \alpha+(N S w \alpha)^{\prime}=\omega^{2} \xi
$$

and the first order equation

$$
\frac{N S^{2}}{r^{2}} w^{2} \alpha+\frac{S}{r^{2}}\left(w^{\prime} \xi-w \xi^{\prime}\right)=\frac{1}{2} \omega^{2} \alpha .
$$

In addition, the Gauss constraint gives

$$
\omega\left\{\left(\frac{r^{2}}{S} \alpha\right)^{\prime}-2 w \xi\right\}=0 .
$$

The following observation simplifies the further discussion of this type of perturbations considerably. If we multiply (6) with $w$ and take the background Yang-Mills equation

$$
w^{\prime \prime}=\frac{N S^{2}}{r^{2}} w\left(w^{2}-1\right)
$$

into account, the left hand side of (6) becomes a total derivative of the left hand side of (7), multiplied by $r^{2} / S$. Introducing the quantities

$$
\chi=\frac{r^{2}}{2 S} \alpha, \quad \gamma^{2}=\frac{2 N S^{2}}{r^{2}}
$$


we find from (6)-(8):

$$
\begin{gathered}
\left(w^{2} \gamma^{2} \chi+w^{\prime} \xi-w \xi^{\prime}\right)^{\prime}=\omega^{2} w \xi, \\
w^{2} \gamma^{2} \chi+w^{\prime} \xi-w \xi^{\prime}=\omega^{2} \chi, \\
\omega\left(\chi^{\prime}-w \xi\right)=0 .
\end{gathered}
$$

When $\omega=0$, there is a residual $U(1)$ gauge symmetry of these equations:

$$
\xi \rightarrow \xi+w \Omega, \quad \chi \rightarrow \chi+\Omega^{\prime} / \gamma^{2},
$$

where $\Omega$ is a function of $\rho$. This will be of crucial importance.

\section{The number of odd-parity sphaleron instabilities}

In this section we prove that the number of negative $\left(\omega^{2}<0\right)$ modes of the system (11)-(13) for the $n$-th BK solution, as well as for the $n$-th EYM black hole, is exactly $n$. We call this type of instabilities sphaleron-like, because of their similarity with the flat spacetime Yang-Mills-Higgs sphaleron instabilities [7].

For $\omega \neq 0$, Eq.(11) is a consequence of (12) and (13), i.e.,

$$
\begin{gathered}
w \xi^{\prime}-w^{\prime} \xi=\left(w^{2} \gamma^{2}-\omega^{2}\right) \chi, \\
\chi^{\prime}=w \xi .
\end{gathered}
$$

As a technical tool we keep equation (16) also for $\omega=0$ and call it strong Gauss constraint; it plays the role of a gauge fixing condition. It suffices to determine the number of $\omega^{2}<0$ modes of the system (15), (16). (The role of the Gauss constraint is discussed more extensively in [10]).

We begin our analysis by eliminating $\xi$, with the result

$$
-\phi^{\prime \prime}+\left(\frac{1}{2} \gamma^{2}\left(w^{2}+1\right)+2\left(\frac{w^{\prime}}{w}\right)^{2}\right) \phi=\omega^{2} \phi,
$$

where $\phi=\chi / w$. For a smooth function $w$ without zeros this would be a regular Schrödinger equation with a purely positive spectrum $\left(\omega^{2} \geq 0\right)$. This suggests that the number of unstable modes of the $n$-th equilibrium solution is indeed related to the number $n$ of nodes of $w$.

Since the differential equation (17) has singularities at the node positions of $w$, we try to pass to a "dual" regular Schrödinger equation, for which the number of bound states can be read off. How to achieve this is suggested by the following considerations.

First, we note that Eq.(17) has obvious $\omega=0$ solutions which are just pure gauge modes (see (14)),

$$
\phi_{0}=\Omega^{\prime} / w \gamma^{2},
$$

satisfying the strong Gauss constraint (16) with $\xi=w \Omega$ :

$$
\left(\frac{\Omega^{\prime}}{\gamma^{2}}\right)^{\prime}=w^{2} \Omega \text {. }
$$


Any such solution allows us to factorize the differential operator in (17):

$$
-\frac{d^{2}}{d \rho^{2}}+\left(\frac{1}{2} \gamma^{2}\left(w^{2}+1\right)+2\left(\frac{w^{\prime}}{w}\right)^{2}\right)=-\frac{d^{2}}{d \rho^{2}}+\frac{\phi_{0}^{\prime \prime}}{\phi_{0}}=Q^{+} Q^{-},
$$

with

$$
Q^{ \pm}=\mp \frac{d}{d \rho}-\frac{\phi_{0}^{\prime}}{\phi_{0}}
$$

Using (19) we find

$$
Q^{ \pm}=\mp \frac{d}{d \rho}+\frac{w^{\prime}}{w}+w^{2} Z
$$

where

$$
Z=-\Omega \Lambda, \quad \Lambda=\gamma^{2} / \Omega^{\prime}
$$

For later use we note that (19) is equivalent to

$$
\Lambda^{\prime} / \Lambda=w^{2} Z
$$

and thus $Z$ satisfies the nonlinear differential equation

$$
Z^{\prime}=w^{2} Z^{2}-\gamma^{2}
$$

With a standard reduction, the most general solution of the second order linear equation (19) is

$$
\Omega=c_{2} \tilde{\Lambda}+\tilde{\Omega}\left(c_{1}+c_{2} \int_{0}^{\rho} w^{2} \tilde{\Lambda}^{2} d \rho\right)
$$

where $\tilde{\Omega}$ is a special solution, $\tilde{\Lambda}=\gamma^{2} / \tilde{\Omega}^{\prime}$, and $c_{1}, c_{2}$ are real constants. This gives immediately for $Z$ in (23)

$$
Z=-\tilde{\Omega} \tilde{\Lambda}-\frac{\tilde{\Lambda}^{2}}{c_{1} / c_{2}+\int_{0}^{\rho} w^{2} \tilde{\Lambda}^{2} d \rho} .
$$

Since the differential equation $(\sqrt{17})$ is, for any $\Omega$ in (26), identical to

$$
Q^{+} Q^{-} \phi=\omega^{2} \phi
$$

we are invited to pass from $\phi$ to

$$
\psi=Q^{-} \phi
$$

For $\omega \neq 0$ this has the unique inverse

$$
\phi=\frac{1}{\omega^{2}} Q^{+} \psi
$$

and by applying $Q^{-}$we obtain the "dual" eigenvalue equation

$$
Q^{-} Q^{+} \psi=\omega^{2} \psi
$$

The differential operator on the left is

$$
Q^{-} Q^{+}=-\frac{d^{2}}{d \rho^{2}}+U
$$


with

$$
U=\frac{1}{2} \gamma^{2}\left(3 w^{2}-1\right)+2\left(w^{2} Z\right)^{\prime} .
$$

In this potential the nodes of $w$ are no more dangerous and we have the chance that (31) becomes a "regular" Schrödinger equation if $\Omega$ in (26) is chosen appropriately.

Before addressing this problem, we determine the zero-energy solutions of (31). These satisfy $Q^{+} \psi_{0}=0$, i.e.,

$$
-\frac{\psi_{0}^{\prime}}{\psi_{0}}=\frac{w^{\prime}}{w}+w^{2} Z=\frac{\phi_{0}^{\prime}}{\phi_{0}}
$$

thus

$$
\psi_{0}=\frac{1}{\phi_{0}}=w \Lambda=w \frac{\tilde{\Lambda}}{c_{1}+c_{2} \int_{0}^{\rho} w^{2} \tilde{\Lambda}^{2} d \rho} .
$$

An alternative form is obtained from (24), (35):

$$
\psi_{0}=w \exp \left(\int_{\rho_{0}}^{\rho} w^{2} Z d \rho\right) \text {. }
$$

This looks good, because the factor $w$ would count the number of nodes of this zeroenergy solution if the second factor on the right hand side could be chosen to be everywhere regular.

A detailed discussion of the function $Z$ in (27), whereby $\tilde{\Omega}$ is a special solution of the linear equation (19), will be given elsewhere. Here we only state that one can choose $\tilde{\Omega}$ such that

$$
\tilde{\Omega}=\frac{\text { const }}{r^{2}}+O(1) \text { as } r \rightarrow 0, \quad \tilde{\Omega}=\frac{1}{r^{2}}+O\left(\frac{1}{r^{3}}\right) \text { as } r \rightarrow \infty
$$

for the BK solitons, and

$$
\tilde{\Omega}=x+O\left(x^{2}\right) \text { as } x \rightarrow 0, \quad \tilde{\Omega}=\text { const } x+O(1) \text { as } x \rightarrow \infty
$$

for the EYM black holes, where $x=\left(r-r_{h}\right) / r_{h}, r_{h}$ being the Schwarzschild coordinate of the horizon.

For the regular solutions, one obtains from (37), (27) (with an appropriate constant $c_{2} / c_{1}$ in (27)) that $Z$ exists everywhere on the half-line with the following behavior near $\rho=0, \infty$ :

$$
Z=\frac{1}{\rho}+O(\rho) \text { as } \rho \rightarrow 0, \quad Z=-\frac{2}{\rho^{2}}+O\left(\frac{\ln (\rho)}{\rho^{3}}\right) \text { as } \rho \rightarrow \infty .
$$

This assures that the potential $U$ in (33) is everywhere bounded. Indeed, the first term for $U$ behaves as $2 / \rho^{2}$ for $\rho \rightarrow 0$, and this singularity is exactly canceled by the second term in $U$ involving the function $Z$.

In summary, the "dual" equation (31) can be chosen such that it becomes an $S$-wave Schrödinger equation with an everywhere bounded potential. In addition (39) assures the following behavior for the zero-energy solution $\psi_{0}$ :

$$
\psi_{0} \sim \rho \text { as } \rho \rightarrow 0, \quad \psi_{0} \sim \frac{1}{\rho^{2}} \text { as } \rho \rightarrow \infty
$$


which implies that $\psi_{0} \in L^{2}[0, \infty)$. A well-known theorem ensures then that the number of nodes of $\psi_{0}$, which is $n$ for the $n$-th BK soliton solution, is also equal the the number of bound states of (31), and thus equal to the number of sphaleron-like instabilities.

Similarly, for black holes, the special solution (38), together with an appropriate choice of $c_{2} / c_{1}$ in (27), ensure that $Z$ has the following behavior near $\rho= \pm \infty$ :

$$
Z=-\frac{1}{f^{2}\left(r_{h}\right) \rho}+O\left(\frac{1}{\rho^{2}}\right) \text { as } \rho \rightarrow-\infty, \quad Z \rightarrow-\frac{2}{\rho^{2}}+O\left(\frac{\ln (\rho)}{\rho^{3}}\right) \text { as } \rho \rightarrow \infty .
$$

This yields

$$
\psi_{0} \sim \frac{1}{\rho} \text { as } \rho \rightarrow-\infty, \quad \psi_{0} \sim \frac{1}{\rho^{2}} \text { as } \rho \rightarrow \infty,
$$

thus $\psi_{0} \in L^{2}(-\infty, \infty)$, implying that for the $n$-th EYM black hole (independent of its event horizon size) there exist precisely $n$ unstable odd-parity modes.

\section{Concluding remarks}

Looking back at the proof, the following elements have been crucial. Thanks to the residual gauge symmetry (14) we know zero energy solutions of the original differential equation (17) and we can thus factorize the differential operator. We can choose this factorization such that the "supersymmetric partner" is a well-behaved Schrödinger equation which has a normalizable zero-energy solution. For this solution, we can determine the number of zeros and hence read off the number of bound states.

Unfortunately, because $\eta$ in Eq.(3) remains invariant under the residual gauge transformation, this method does not work for the even-parity gravitational instabilities, whose number is presumably the same.

\section{Acknowledgments}

This work was supported by the Swiss National Science Foundation and by the Tomalla Foundation.

\section{References}

[1] R. Bartnik, J. McKinnon, Phys. Rev. Lett. 61 (1988) 141.

[2] M.S. Volkov, D.V. Gal'tsov, Pis'ma Zh. Eksp. Teor. Fiz. 50 (1989) 312

[JETP Lett. 50 (1990) 345]; Sov. J. Nucl.Phys. 51 (1990) 747;

H.P. Kunzle, A.K.M. Masood-ul-Alam, J. Math. Phys. 31 (1990) 928;

P. Bizon, Phys. Rev. Lett. 64 (1990) 2644.

[3] N. Straumann, Z.H. Zhou, Phys. Lett. B 237 (1990) 353;

N. Straumann, Z.H. Zhou, Phys.Lett. B 243 (1991) 53.

[4] Z.H. Zhou, N. Straumann, Nucl. Phys. B 369 (1991) 180; 
[5] Z.H. Zhou, Helv. Phys. Acta 65 (1992) 767.

[6] N.S.Manton, Phys.Rev. D 28 (1983) 2019;

F.R.Klinkhamer, N.S.Manton, Phys.Rev. D 30 (1984) 2212.

[7] D.V. Gal'tsov, M.S. Volkov, Phys. Lett. B 273 (1991) 255.

[8] M.S. Volkov, Phys. Lett. B 328 (1994) 89; Phys. Lett. B 334 (1994) 40.

[9] O. Brodbeck, N. Straumann, Phys. Lett. B 324 (1994) 309.

[10] O. Brodbeck, N. Straumann, preprint ZU-TH 38/94, gr-qc/9411058.

[11] M.S. Volkov, D.V. Gal'tsov, Phys.Lett. B 341 (1995) 279.

[12] G. Lavrelashvili, D. Maison, Phys.Lett. B 343 (1995) 214.

[13] J.A. Smoller, A.G. Wasserman, S.T. Yau, and J.B. McLeod, Comm.Math.Phys. 143 (1991) 115.

[14] J.A. Smoller, A.G. Wasserman, Comm.Math.Phys. 151 (1993) 303; ibid. 154 (1993) 377.

[15] P. Breitenlohner, P. Forgacs, D. Maison, Comm. Math. Phys. 163 (1994) 141.

[16] P. Boschung, O. Brodbeck, F. Moser, N. Straumann, and M.S. Volkov, Phys.Rev. D 50 (1994) 3842. 